\title{
Search for the standard model Higgs boson in the $\mathbf{Z}$ boson plus a photon channel in CMS
}

\section{Chia Ming, Kuo for the CMS Collaboration*}

Department of Physics, National Central University, Chung-li, Taiwan

E-mail: chia-ming.kuo@cern.ch

\begin{abstract}
A search for the standard model Higgs boson decaying to a $\mathrm{Z}$ boson plus a photon with subsequent decay of the $\mathrm{Z}$ boson to a final state containing two leptons, $\mathrm{H} \rightarrow \mathrm{Z} \gamma \rightarrow 1^{+} 1^{-} \gamma$, is presented. Results are based on data collected at center-of-mass energies of $7 \mathrm{TeV}$ and $8 \mathrm{TeV}$, corresponding to integrated luminosities of $5.0 \mathrm{fb}^{-1}$ and $19.6 \mathrm{fb}^{-1}$, respectively. At the Higgs boson mass of $125 \mathrm{GeV} / \mathrm{c}^{2}$ the observed and expected limits are within one order of magnitude of the standard model prediction.
\end{abstract}

The European Physical Society Conference on High Energy Physics -EPS-HEP2013 18-24 July 2013

Stockholm, Sweden

\footnotetext{
* Speaker.
} 


\section{Introduction}

The standard model (SM) successfully explains the vast majority of the existing experimental data. The discovery of a Higgs boson [1,2] provides a gateway for exploring physics beyond the SM. The properties of the Higgs boson are precisely predicted by the SM for a given mass value. Within the SM, the partial width for the $\mathrm{H} \rightarrow \mathrm{Z} \gamma$ decay channel is small, resulting in a rather small branching ratio between $0.11 \%$ and $0.25 \%$ in the mass range of $120<m_{\mathrm{H}}<160 \mathrm{GeV}$. However, it is of particular interest to measure $\mathrm{H} \rightarrow \mathrm{Z} \gamma$ decay because it is induced by loops of heavy charged particles, just as the $\mathrm{H} \rightarrow \gamma \gamma$ decay, and thus sensitive to physics beyond the SM [4, 5, 6, 7].

This proceeding reports the search for a Higgs boson in the $\mathrm{H} \rightarrow \mathrm{Z} \gamma$ final state at the LHC [8]. The analysis strategy is to select events with two oppositely charged leptons (electrons or muons only) and an energetic photon. The effective mass resolution is about 1-3\%. The events are further categorized into different classes to improve the sensitivity of the search. The main SM background components are the irreducible contribution from the SM $Z \gamma$ production, and the reducible backgrounds from final-state-radition in Drell-Yan or $\mathrm{Z}$ decays, and $\mathrm{Z}+\mathrm{jets}$, where a jet is misidentified as a photon. The data sample recorded by the CMS experiment [3] corresponds to an integrated luminosity of $5.0 \mathrm{fb}^{-1}$ at $\sqrt{s}=7 \mathrm{TeV}$ and $19.6 \mathrm{fb}^{-1}$ at $\sqrt{s}=8 \mathrm{TeV}$ in proton-proton collisions.

\section{Event selection, event classes, and signal and background modeling}

Events are recorded by at least one of the dielectron or dimuon triggers. Both electrons and muons are required to have $p_{T}>20$ and $10 \mathrm{GeV}$, for higher and lower $p_{T}$ lepton, respectively. Electrons (muons) and the photon in the pseudorapidity range $|\eta|<2.5(|\eta|<2.4)$ are considered. Photons in the transition region between the barrel and endcap, $1.44<|\eta|<1.57$, of the electromagnetic calorimeter are excluded. All particles are required to be isolated from hadronic activity in the event. The invariant mass of the lepton pair is required to be greater than $50 \mathrm{GeV}$. The ratio of the photon transverse energy to the invariant mass of the $l^{+} l^{-} \gamma$ system, $m_{l l \gamma}$, must be greater than $15 / 110$ to suppress backgrounds due to misidentification of photons without significant loss in signal sensitivity and without introducing a bias in the $m_{l l \gamma}$ spectrum. Photons are required to be separated from the lepton by $\Delta R(l, \gamma)>0.4$. The remaining final-state radiation events are rejected by requiring the sum of $m_{l l \gamma}$ and $m_{l l}$ to be at least $185 \mathrm{GeV}$. Jets are reconstructed using the anti-k clustering algorithm [9] with distance parameter $\Delta R=0.5$. Calibrated and selected jets are required to have $p_{T}>30 \mathrm{GeV}$ and $|\eta|<4.7$ and to be separated by at least 0.5 in $\Delta R$ from selected leptons and photons.

The sensitivity of the search can be enhanced by $20-40 \%$ by subdividing the selected events into classes according to indicators of mass resolution and signal-to-background ratio and combining the results of a search in each class. The events are classified according to the pseudorapidity of the leptons, the pseudorapidity of the photon and the shower shape of the photon for events with the two leptons in the barrel. A further class of events includes any event passing a dijet tag defined to select Higgs bosons produced by the vector-boson fusion (VBF) process. Events in which a Higgs boson is produced by VBF have two forward jets, originating from the two scattered quarks, and an expected signal-to-background ratio more than an order of magnitude greater than events in other classes defined by lepton and photon properties. The requirements for the dijet-tagged 
event class include the pseudorapidity separation between two selected jets, their invariant mass, the difference between the average pseudorapidity of the two jets and the pseudorapidity of the $Z \gamma$ system, and the difference in azimuthal angle between the $Z \gamma$ system and the dijet system. The additional classification of events into a dijet-tagged class improves the sensitivity of the search by $10-15 \%$. Events in different event classes are mutually exclusive.

The background model is obtained by fitting the observed $m_{l l \gamma}$ dsitribution in each event class over the range $100<m_{l l \gamma}<190 \mathrm{GeV}$. A thorough bias study was performed to ensure the bias introduced on the limit of the signal strength measurement is smaller than a fifth of the background statistical uncertainty. The signal description is obtained from MC simulation.

\section{Results}

The expected and observed limits are shown in Fig. 1. No excess above the SM predictions has been found. The observed and expected limits for $m_{l l \gamma}$ at $125 \mathrm{GeV}$ are within one order of magnitude of the SM prediction. Models predicting $\sigma(p p \rightarrow \mathrm{H}) \times \mathrm{B}(\mathrm{H} \rightarrow \mathrm{Z} \gamma)$ to be larger than one order of magnitude of the SM prediction, for most of the $125-157 \mathrm{GeV}$ mass range, are excluded.
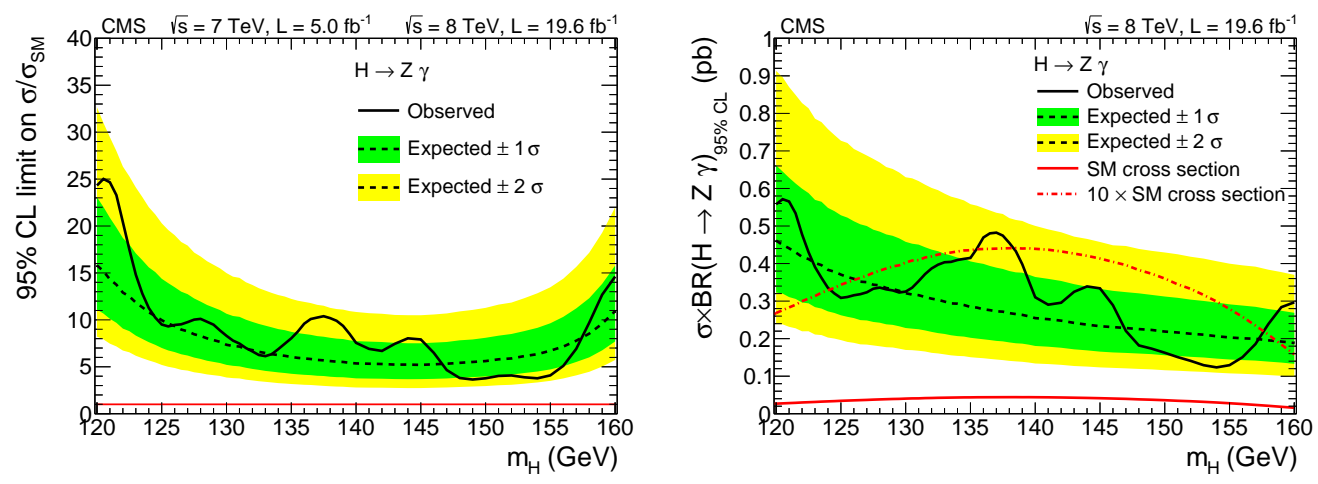

Figure 1: (left) Observed (solid) and expected (dashed) 95\% CL upper limits on the ratio of the production cross section to the SM expectation for the Higgs boson. (right) Exclusion limit on the inclusive cross section.

\section{References}

[1] CMS Collaboration, Phys. Lett. B 716 (2012) 30

[2] ATLAS Collaboration, Phys. Lett. B 716 (2012) 1

[3] CMS Collaboration, JINST 3 (2008) S08004

[4] M. Carena, I. Low, and C. E. M. Wagner, JHEP 8 (2012) 60

[5] C.-W. Chiang and K. Yagyu, Phys. Rev. D 87 (2013) 33003

[6] I. Low, J. Lykken, and G. Shaughnessy, Phys. Rev. D 84 (2011) 35027

[7] C.-S. Chen, C.-Q. Geng, D. Hung, and L.-H. Tsai, Phys. Rev. D 87 (2013) 75019

[8] CMS Collaboration, Phys. Lett. B 726 (2013) 587

[9] M. Cacciari, G. P. Salam, and G. Soyez, JHEP 04 (2008) 063 